\title{
Expression pattern of circulating long non-coding RNA GAS5 as a novel biomarker in non-small cell lung cancer patients
}

Natasa Vesovic ${ }^{1}$, Natasa Tosic ${ }^{2}$, Teodora Karan Djurasevic ${ }^{2}$, Zoran Andric ${ }^{3}$, Darko Zdravkovic ${ }^{3}$, Sonja Pavlovic ${ }^{2}$, Dragana Jovanovic ${ }^{4}$

\author{
${ }^{1}$ Clinic for Chest Surgery, Medical Military Academy, Belgrade, Serbia \\ ${ }^{2}$ Institute of Molecular Genetics and Genetic Engineering, University of Belgrade, \\ Belgrade, Serbia \\ ${ }^{3}$ Clinics for Oncology, University Hospital Medical Centre Bezanijska Kosa, Belgrade, \\ Serbia \\ ${ }^{4}$ University Hospital of Pulmonology, Clinical Centre of Serbia, Belgrade, Serbia
}

Submitted: 8 April 2020

Accepted: 13 June 2020

Arch Med Sci

DOI: https://doi.org/10.5114/aoms.2020.98815

Copyright (c) 2020 Termedia \& Banach

\section{Abstract}

Introduction: The finding that long noncoding RNAs (IncRNAs) originating from tumor cells could be found in general circulation has prompted the idea to use IncRNAs as noninvasive diagnostic biomarkers of particular diseases. In this study we explored the expression pattern of circulating GAS5 (growth arrest-specific transcript 5) IncRNA in non-small cell lung cancer (NSCLC) patients and its association with clinicopathological characteristics. Material and methods: Expression pattern of circulating GAS5 was analyzed in 58 plasma samples of NSCLC patients, and 15 healthy controls. Quantitative assessment was performed using the real-time PCR method and TaqMan chemistry.

Results: Circulating GAS5 expression level in NSCLC patients was not significantly decreased compared to control samples $(p=0.081)$. Statistically significant difference in GAS5 expression was found in relation to TNM stage of the tumor $(p<0.001)$, decreasing with progression of the tumor stage. Lower GAS5 expression was detected in patients with larger tumors $(p=0.006)$, and in patients with lymph node metastasis $(p=0.001)$. Receiver operating characteristic curve analysis was used to evaluate the diagnostic potential of circulating GAS5 expression, showing the highest predictive power in distinguishing between stage III/IV patients and control samples (AUC $=0.8$; sensitivity $53 \%$, specificity $93 \%$ ), and also for separating patients between TNM stage I/II and stage III/IV (AUC = 0.82; sensitivity $73 \%$, specificity $79 \%$ ). Conclusions: Our study suggests that decreased expression of circulating GAS5 is closely related to the tumor size and TNM stage. Therefore the measurement of GAS5 expression level in plasma could be a promising noninvasive diagnostic molecular biomarker in NSCLC patients.

Key words: non-small cell lung cancer, plasma RNA, long non-coding RNA, GAS5.

\section{Introduction}

Non-small cell lung cancer (NSCLC) is the most common type of lung cancer, which can be found in about $85 \%$ of cases [1]. There are three

\author{
Corresponding author: \\ Natasa Tosic, PhD \\ Institute of Molecular \\ Genetics \\ and Genetic Engineering \\ University of Belgrade \\ Belgrade, Serbia \\ E-mail: natasa.tosic@imgge. \\ bg.ac.rs
}


histological subtypes of NSCLC: adenocarcinoma (AC), squamous cell carcinoma (SCC) and large cell carcinoma (LCC) [2]. Regardless of the great improvement in the treatment of NSCLC, including targeted chemotherapy and radiotherapy, five-year survival of these patients is only $15 \%$ [3]. Treatment approach depends primarily on the stage of the disease, and, as one of the main features of NSCLC is its asymptomatic nature, this type of cancer is usually diagnosed in an advanced stage, when conventional therapy is not efficient. Therefore, the mortality among NSCLC patients remains high [4].

In the current research of NSCLC there is a constant need for the discovery of novel biomarkers with high specificity and sensitivity, which could be used in early diagnosis. Also, new biomarkers could serve as targets for the application of new therapy. The discovery that nucleic acids originating from cancer cells can be found in the peripheral circulation of cancer patients has had a major impact towards development of noninvasive techniques for the detection of new molecular biomarkers that can be used in diagnosis of different types of cancers, including NSCLC [5].

In recent years, much attention has been focused on long noncoding RNAs (IncRNAs) and their role in cancer pathology [6-9]. LncRNAs are defined as transcribed RNA molecules with length greater than 200 nucleotides. They are not translated into protein, but are involved in gene regulation and genome activity. Also, they represent an important element of epigenetic regulation of the nuclear and mitochondrial genome, and the disturbances in IncRNA functioning underlie a number of diseases, not just cancer [10,11]. Consequently, they regulate main cell processes such as differentiation, proliferation and the cell cycle $[12,13]$. LncRNA originating from the primary tumor cells can be detected in the plasma of cancer patients. Expression pattern of numerous IncRNAs has been studied in NSCLC patients $[14,15]$. Among them GAS5 (growth arrest-specific transcript 5) could be the most promising new molecular biomarker for distinguishing NSCLC patients from noncancerous subjects $[16,17]$.

The GAS5 gene, located at the 1q25 locus, is approximately 650 base pairs in length and it is organized in 12 exons and 11 introns. Introns encode 10 box C/D snoRNAs (small nucleolar RNAs) that are involved in the methylation process, and therefore in epigenetic regulation. Exons encode few splice variants of GAS5 mRNA, but due to the presence of a stop codon, none of them produce protein, and transcripts are degraded through the nonsense mediated decay (NMD) pathway [18]. The expression level of
GAS5 is increased during growth arrest by serum deprivation [19]. On the other hand, GAS5 is down-regulated in many types of cancer including NSCLC, indicating tumor suppressor function of this IncRNA [20]. It has been proven that in creased expression of GAS5 induces apoptosis in vitro, and reduces tumor growth in vivo [21]. Also, it was shown that up-regulation of GAS5 in NSCLC suppresses cell proliferation, growth and invasion of tumor cells [22, 23].

In the present study we examined the expression pattern of circulating GAS5 in the plasma samples of NSCLC patients and in healthy individuals, and investigated the association between GAS5 expression level and patients' clinicopathological features. Also, we evaluated whether GAS5 expression level could serve as novel, noninvasive molecular biomarker in NSCLC diagnosis.

\section{Material and methods}

\section{Patients}

Samples of 58 patients diagnosed with NSCLC (median 65 years, range 45-81) and 15 healthy controls were collected from UHMC Bezanijska Kosa, Belgrade, from May 2018 to March 2019. Samples were collected from the patients before any kind of treatment. The study was approved by the Ethics Committee of the UHMC Bezanijska Kosa, Belgrade, and all of the participants signed an informed consent form according to the principles of the Declaration of Helsinki.

All patients underwent diagnostic procedures that included bronchoscopy, CT scan of the chest and abdomen, skeletal scintigraphy and brain MRI. Patients were assigned to the appropriate stage according to the post-surgical pathohistological findings (TNM classification). All of the clinical and pathological data of patients are displayed in Table I.

\section{Plasma preparation and RNA extraction}

For each patient and healthy controls blood samples were collected in $9 \mathrm{ml}$ EDTA anti-coagulation tubes and were kept at $4^{\circ} \mathrm{C}$ and then transported to the Institute of Molecular Genetics and Genetic Engineering for further processing. Plasma was separated within $4 \mathrm{~h}$ after collection using a two-step centrifugation protocol. First, blood samples were centrifuged for $10 \mathrm{~min}$ at $1900 \mathrm{~g}$, at $4^{\circ} \mathrm{C}$, and then a second centrifugation step was performed to eliminate any cell debris (10 $\mathrm{min}$ at $16000 \mathrm{~g}$, at $\left.4^{\circ} \mathrm{C}\right)$. Supernatant was collected in aliquots of $500 \mu \mathrm{l}$ and stored at $-80^{\circ} \mathrm{C}$.

Total RNA extraction was performed using the miRNeasy Serum/Plasma Kit (Qiagen, Germany) and vacuum manifold QIAvac24 Plus (Qiagen, Germany), with slight modifications of the manufac- 
turer's protocol. The volume of starting material was raised to $500 \mu \mathrm{l}$ as it was suggested by the NIH Extracellular RNA Communication Consortium [24]. The volume ratio between plasma, Qiazol LR and chloroform was kept constant at $1: 5: 1$, and additional washing steps were performed when using RPE buffer and 80\% ethanol. Finally, double elution was performed using $30 \mu \mathrm{l}$ of RNase-free water. The final RNA yield ranged from 200-750 ng, per $500 \mu$ l of plasma.

\section{Quantitative reverse transcription polymerase chain reaction (qRT-PCR)}

Total RNA (400 ng) was reverse transcribed into cDNA using RevertAid Reverse Transcriptase (Thermo Scientific). Real time-PCR was performed with the 7900 HT Fast Real-Time PCR System (Applied Biosystems). We performed PCR using $1 \mu \mathrm{l}$ of cDNA (20 ng RNA equivalent) with TaqMan Universal Master Mix II (Applied Biosystems), and predesigned (ready-to-go) TaqMan Gene Expression Assay for GAS5 (Hs03464472_m1) and for GAPDH (Hs99999905_m1) as an endogenous control (Thermo Fisher Scientific). All samples were run in duplicate. The PCR reaction was performed at $95^{\circ} \mathrm{C}$ for $10 \mathrm{~min}$, followed by 50 cycles of $95^{\circ} \mathrm{C}$ for $15 \mathrm{~s}$, and $60^{\circ} \mathrm{C}$ for $1 \mathrm{~min}$. Relative quantification analysis was performed using the comparative $d d C t$ method, using healthy controls as a calibrator, meaning $d d C t=d C t_{\text {sample }}-d C t_{\text {healthy control (median). }}$.

\section{Statistical analysis}

The data were descriptively reported as medians with range, means $\pm \mathrm{SD}$, or as absolute numbers with percentages. Differences in continuous variables were analyzed using the Mann-Whitney $U$ test for distribution between two groups. Receiver operating curve (ROC) curve analysis was done to evaluate predictive power of GAS5 expression level in diagnosis and staging of NSCLC patients.

The statistical analyses were performed using the SPSS computer software 21.0 (IBM). For all analyses, the $p$ values were 2 -tailed, and $p<0.05$ was considered statistically significant.

\section{Results}

\section{GAS5 expression and clinicopathological features}

The expression level of GAS5 was evaluated in a cohort of 58 NSCLC patients and in 15 healthy individuals. The median expression level of GAS5 among patients was 0.65 (range 0.00-3.76), which was not significantly lower compared to the expression level found among healthy individuals (median $1.00 \pm 1.30$, range $0.15-4.31$, $p=0.081)$. Also, GAS5 expression level in the patients' plasma samples was not significantly correlated with age, gender, smoking status or histology type (Table I). On the other hand, comparing GAS5 expression between the patients with different tumor sizes (smaller and larger than $3 \mathrm{~cm}$ ) we detected a statistically significantly lower value in patients with larger tumors $(p=$ 0.006) (Figure $1 \mathrm{~A}$ ).

We also detected a statistically significant difference in GAS5 expression in relation to TNM stage of the tumor (Table I). When analyzing individual associations between TNM stages, we detected the largest statistical difference between stage III patients and all other stages (Figure $1 \mathrm{~B}$ ). When we analyzed GAS5 expression in NSCLC patients at different $T$ stages, we observed that GAS5 expression decreased slowly as the T-stage increased. Significant differences were detected between T1 and T3/T4 stages ( $p=0.016$ and $p=0.003$, respectively) (Figure $1 \mathrm{C}$ ).

Significant differences in GAS5 expression were detected regarding the presence of lymph node metastasis $(p=0.001)$. Patients positive for these metastasis had much lower expression of GAS5 compared to patients without lymph node involvement (Figure $1 \mathrm{D})$.

\section{Diagnostic potential of GAS5 expression in NSCLC}

We used ROC curve analysis to calculate the predictive power of GAS5 expression to discriminate between NSCLC patients and healthy individuals. In our cohort of patients the value of AUC was 0.65 (95\% Cl: $0.5-0.794)(p=0.081)$, and the sensitivity and specificity were $24 \%$ and $100 \%$, for the cut-off value of 0.135 (Figure $2 \mathrm{~A}$ ).

Compared to healthy control samples, only GAS5 expression detected in stage III patients gave a significant predictive value in ROC curve analysis with AUC $=0.93$ (95\% Cl: 0.842-1.024) $(p<0.001$ ), sensitivity $87 \%$ and specificity $93 \%$ (cut-off value 0.18 ) (Figure 2 B). Also, when we combined stage III and stage IV patients and compared them to control samples, circulating GAS5 expression showed significant predictive value with $\mathrm{AUC}=0.80(95 \% \mathrm{Cl}: 0.668-0.928)$ ( $p=0.001$ ), sensitivity $53 \%$ and specificity $93 \%$ (cut-off value 0.18) (Figure $2 \mathrm{C}$ ).

Next, we evaluated whether based on GAS5 expression we could distinguish NSCLC patients at different TNM stages. Patients with stage I and stage II tumors were compared to patients with stage III or stage IV tumors and ROC curve analysis showed strong predictive power with AUC of 0.82 (95\% Cl: 0.710-0.923) ( $p<0.001), 73 \%$ sensitivity and specificity of $79 \%$ (cut-off value 0.64) (Figure 2 D). 
Natasa Vesovic, Natasa Tosic, Teodora Karan Djurasevic, Zoran Andric, Darko Zdravkovic, Sonja Pavlovic, Dragana Jovanovic

Table I. Association of GAS5 expression with clinicopathological characteristics of patients with NSCLC

\begin{tabular}{|c|c|c|c|}
\hline Variable & $\begin{array}{l}\text { No of patients } \\
\quad(N=58)\end{array}$ & GAS5 expression & $P$-value \\
\hline Gender (\%): & & & 0.347 \\
\hline Male & $41(71)$ & $0.60(0.02-3.76)$ & \\
\hline Female & $17(29)$ & $0.97(0.00-3.19)$ & \\
\hline Age (years) (\%): & & & 0.668 \\
\hline$\leq 65$ & $27(47)$ & $0.65(0.03-3.19)$ & \\
\hline$>65$ & $31(53)$ & $0.65(0.00-3.76)$ & \\
\hline Smoking status (\%): & & & 0.872 \\
\hline Yes & $53(91)$ & $0.65(0.02-3.76)$ & \\
\hline No & $5(9)$ & $0.80(0.00-2.60)$ & \\
\hline Tumor size (\%): & & & 0.006 \\
\hline$\leq 3 \mathrm{~cm}$ & $13(22)$ & $1.58(0.38-3.76)$ & \\
\hline$>3 \mathrm{~cm}$ & $45(78)$ & $0.53(0.00-3.19)$ & \\
\hline Histology type (\%): & & & 0.242 \\
\hline Adenocarcinoma & $28(48)$ & $0.66(0.00-3.76)$ & \\
\hline Squamous carcinoma & $26(45)$ & $0.64(0.05-3.19)$ & \\
\hline Non-microcellular & $4(7)$ & $0.13(0.08-0.68)$ & \\
\hline TNM stage (\%): & & & $<0.001$ \\
\hline 1 & $16(28)$ & $1.22(0.08-3.76)$ & $<0.001$ (I vs. III) \\
\hline $\mathrm{IA}$ & 10 & $2.36(0.38-3.76)$ & $<0.001$ (IA vs. III), 0.036 (IA vs. IV) \\
\hline IB & 6 & $1.00(0.08-3.10)$ & \\
\hline II & $12(21)$ & $1.12(0.41-3.19)$ & $<0.001$ (II vs. III) \\
\hline III & $15(26)$ & $0.09(0.00-1.16)$ & \\
\hline IV & $15(26)$ & $0.63(0.03-2.41)$ & 0.002 (IV vs. III) \\
\hline T stage (\%): & & & 0.016 \\
\hline $\mathrm{T} 1$ & $12(21)$ & $1.91(0.38-3.76)$ & \\
\hline $\mathrm{T} 2$ & $15(26)$ & $0.68(0.03-3.10)$ & \\
\hline $\mathrm{T3}$ & $17(29)$ & $0.57(0.00-3.19)$ & 0.016 (T3 vs. T1) \\
\hline $\mathrm{T} 4$ & $14(24)$ & $0.16(0.03-2.41)$ & 0.003 (T4 vs. T1) \\
\hline Lymph node metastasis: & & & 0.001 \\
\hline Negative & $27(47)$ & $1.20(0.05-3.76)$ & \\
\hline Positive & $31(53)$ & $0.48(0.00-2.41)$ & \\
\hline
\end{tabular}

\section{Discussion}

In recent years, a lot of attention has been given to the study of the role of long noncoding RNAs in different cancers, including non-small lung cancer (NSCLC). The fact that IncRNAs are relatively stable in plasma samples has prompted the idea to use them as noninvasive diagnostic molecular markers of the disease. In this study we investigated the expression pattern of GAS5, IncRNA with a known functional role in NSCLC pathogenesis.

We found that GAS5 expression level was not significantly downregulated in patients compared to healthy individuals. A similar finding was reported by Tan et al. although they detected a difference between stage I patients and healthy 
A

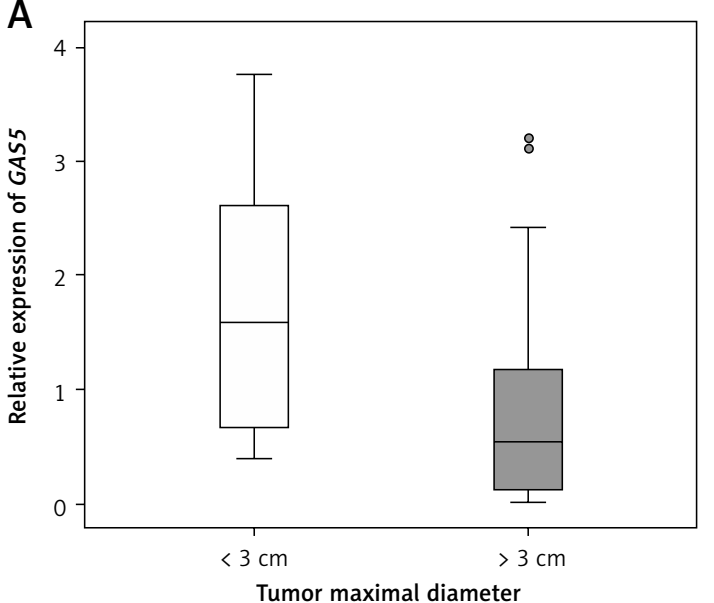

C

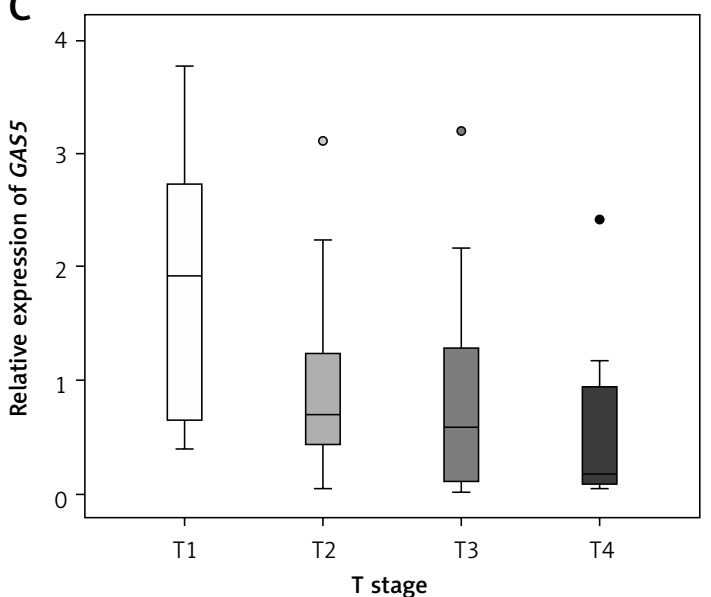

B

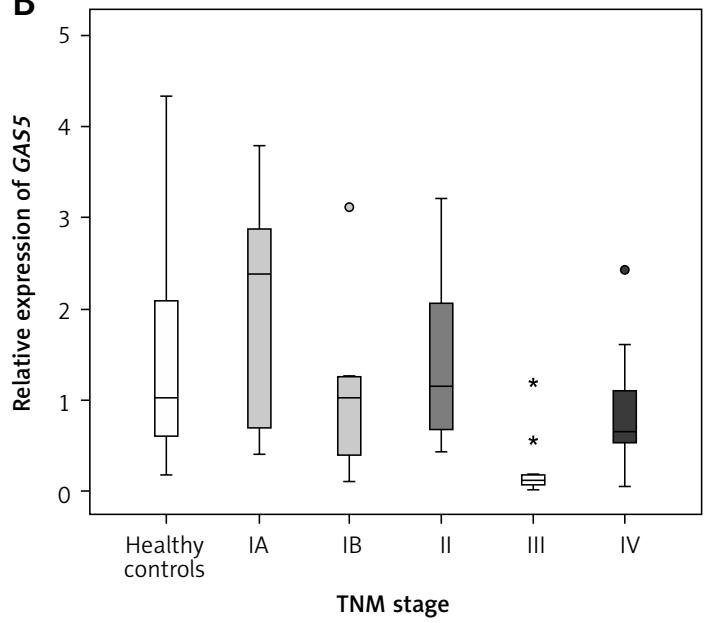

D

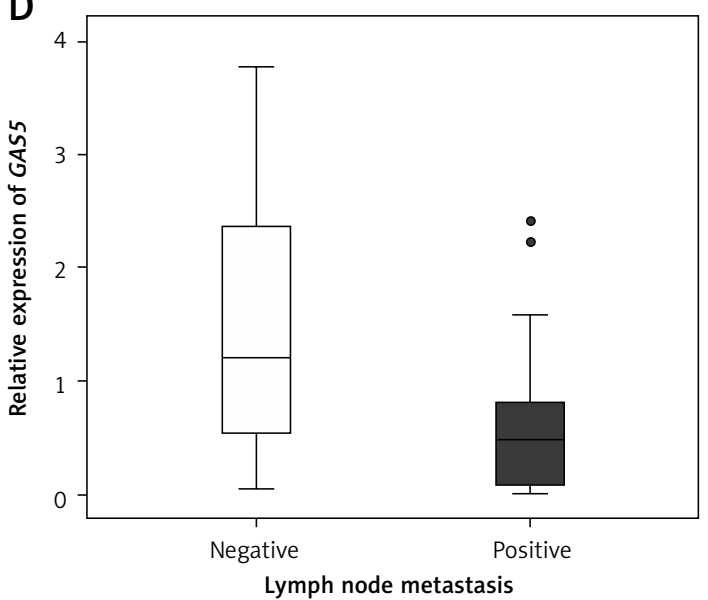

Figure 1. Box-plot presentation of GAS5 expression level in relation to: A - tumor size, B - TNM stage of the tumor, $\mathbf{C}$ - T stage of the tumor, $\mathbf{D}$ - presence of lymph node metastasis

controls, but this difference was marginally significant $(p=0.049)$ [17]. In our cohort of patients we were unable to replicate this finding even when we separated stage I patients into IA and IB stage (Figure $1 \mathrm{~B}$ ). Still, we detected a decrease in GAS5 expression in stage IB compared to stage IA (2.36 vs. 1.00 , Figure $1 \mathrm{~B}$ ) and that value in stage IB is more similar to the one detected in stage II (1.12). Moreover, based on GAS5 expression level we could clearly discriminate patients with stage III tumor from the control group ( $p<0.001)$, and all other TNM stages (Table I). Similar results are reported by two other studies dedicated entirely to analyzing GAS5 expression in plasma samples of NSCLC patients, where a significant decrease in GAS5 expression was detected in later TNM stages $[16,25]$. We assume that uneven values of GAS5 expression, i.e. non-linear decrease through TNM stages detected in previously mentioned studies (as well as in this one), primarily reflect different approaches in the calculation of relative GAS5 expression, and also the overall small number of patients. Also, when determining GAS5 expression in NSCLC patients the origin of the sample should be taken into account. Namely, Tan et al. analyzed paired tissue/plasma samples and found that the decrease in GAS5 expression observed with progression of TNM tumor stages is detected within tissue samples, but not in plasma samples from the same NSCLC patients [17].

Moreover, we found that circulating GAS5 is a great predictor of tumor size, since patients with a tumor larger than $3 \mathrm{~cm}$ had significantly diminished expression compared to patients with a tumor $\leq 3 \mathrm{~cm}$. This result, which was also reported by others, can be interpreted through known tumor-suppressor function of GAS5 [21]. Consistent with this tumor-suppressor function is our finding that GAS5 expression level differed between tumor T- stages, i.e. GAS5 expression level decreased with increasing $T$ stage of the tumor.

GAS5 also plays a role in tumorigenesis by contributing to the formation of metastases [22]. In our cohort, NSCLC patients with regional lymph node metastasis had significantly diminished GAS5 expression in comparison to patients without such metastasis. Recently in in vitro experiments it was found that suppression 
A

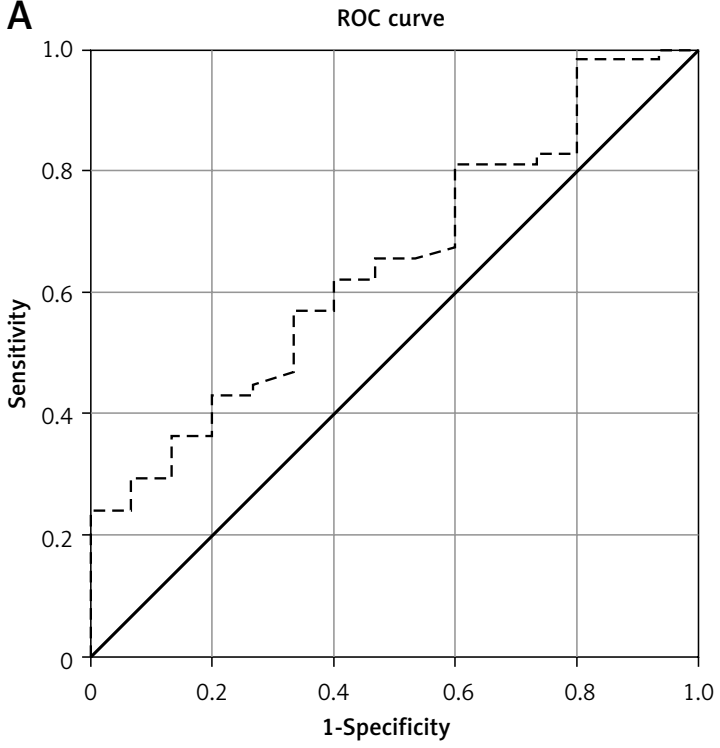

C

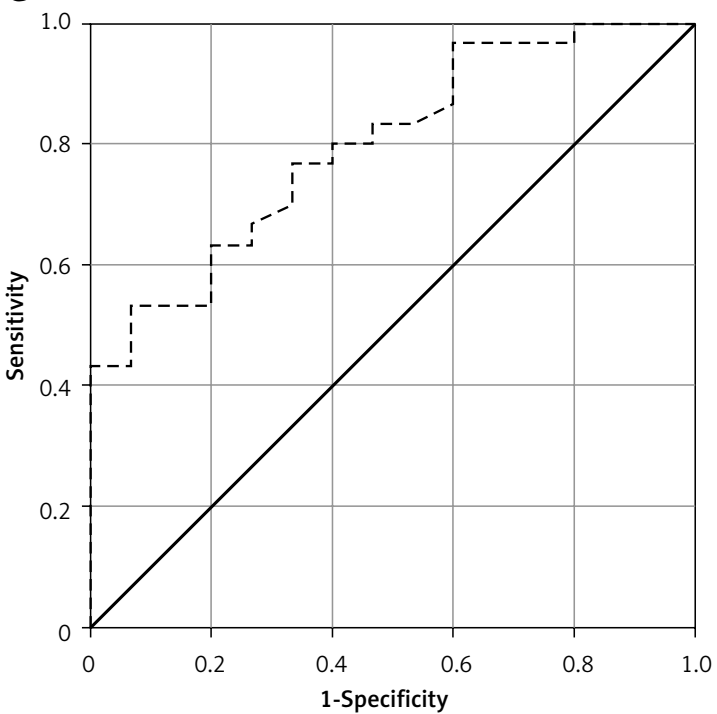

B

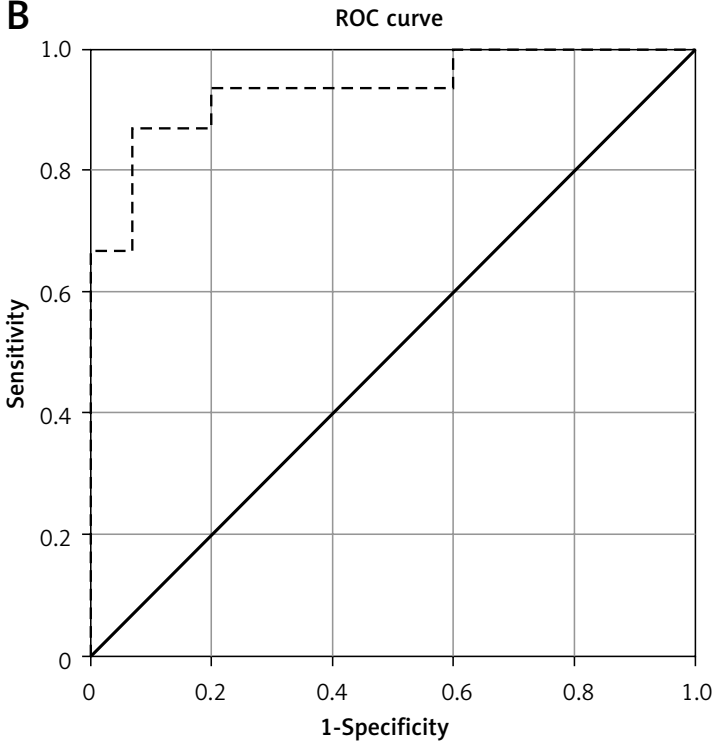

D

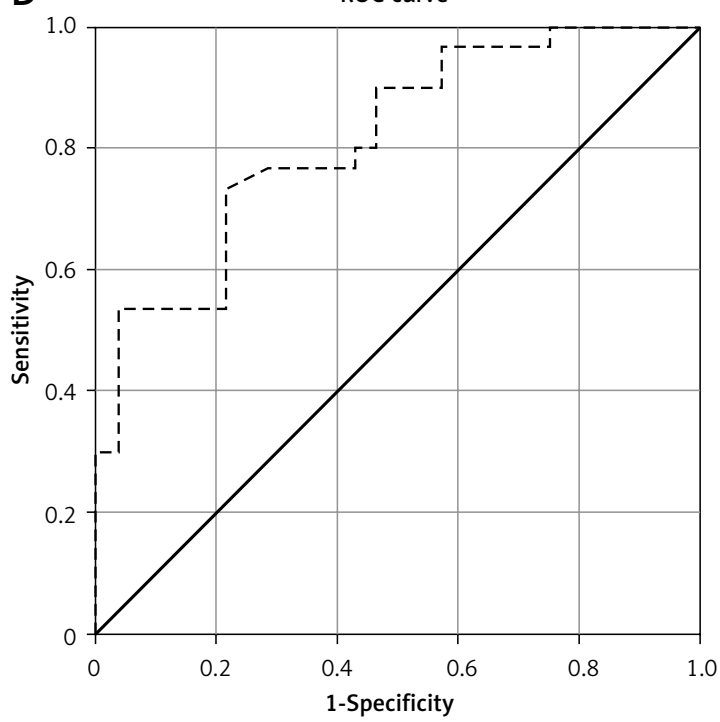

Figure 2. Receiver operating characteristic (ROC) curve for GAS5 expression levels discriminating between: A - whole cohort of NSCLC patients and healthy controls, B - TNM stage III NSCLC patients and healthy controls, C - TNM stage III and IV NSCLC patients and healthy controls, D - TNM stage I/II and stage III/IV NSCLC patients

of GAS5 enables the increase of cell migration and invasion, and in that way contributes to the progression of NSCLC. An even more significant finding of these experiments was that GAS5 expression was observed to be controlled by PTEN (phosphatase and tensin homolog), a tumor-suppressor protein. More precisely, the existence of this GAS5/miR-205/PTEN axis was detected [23]. GAS5 suppresses cell proliferation, migration and invasion through inhibition of miR-205. miR-205 inhibits PTEN expression by interfering with PTEN translational regulation. Inhibition of miR-205 through GAS5 directly leads to overexpression of PTEN and evident tumor suppressor effect. Thus, regulation of the PTEN signaling pathway may serve for application of new targeted NSCLC therapy [23].
Analyzing the potential of GAS5 expression level as a diagnostic tool, we did not observe its importance in distinguishing between early stage tumor patients (stage I/II) and healthy controls. A clear distinction was observed only in stage III patients. In other studies, similar results were reported, but they also reported the diagnostic potential of combined GAS5 expression with tumor markers such as CEA, SCCA and CYFRA21-1, and also with other IncRNAs such as SOX2OT and ANRIL $[16,25]$. It seems that more accurate differential diagnosis can be achieved by combining GAS5 expression level with other markers.

Our study showed that expression of plasma GAS5 was down-regulated in NSCLC patients. Degree of the decrease in GAS5 expression was associated with progression of TNM tumor stage, 
and also with the tumor size and the presence of lymph node metastasis. In addition, GAS5 expression showed significant predictive value for distinguishing between stage III/IV NSCLC patients and control subjects, as well as between patients in different TNM stages. Based on this, we can conclude that GAS5 expression level shows potential to be used as a noninvasive diagnostic molecular marker in NSCLC patients.

Still, this study has a few limitations, involving, primarily, small sample size for both NSCLC and healthy controls. Further investigations on larger numbers of patients would determine the true value of plasma GAS5 expression level as an early prognostic biomarker for NSCLC. We also propose the standardization of methodology used for plasma RNA isolation and real-time-PCR quantification of GAS5, in order to set a "universal" cut-off value that would discriminate between healthy individuals and early stage NSCLC patients. Without such standardization, the introduction of this noninvasive method for the detection of GAS5 as a new biomarker is still far away from everyday clinical practice.

\section{Acknowledgments}

This work was funded by the Ministry of Education, Science and Technological Development, Republic of Serbia (grant number III 41004).

\section{Conflict of Interests}

The authors declare no conflict of interest.

\section{References}

1. Ettinger DS, Akerley W, Bepler G, et al. Non-small cell lung cancer. J Natl Compr Cancer Netw 2010; 8: 740-801.

2. Goldstraw P, Ball D, Jett JR, et al. Non-small-cell lung cancer. Lancet 2011; 378: 1727-40.

3. DeSantis CE, Lin CC, Mariotto AB, et al. Cancer treatment and survivorship statistics. CA Cancer J Clin 2014; 64: 252-71.

4. Rosell R, Bivona TG, Karachaliou N. Genetics and biomarkers in personalisation of lung cancer treatment. Lancet 2013; 382: 720-31.

5. Rapisuwon S, Vietsch EE, Wellstein A. Circulating biomarkers to monitor cancer progression and treatment. Comput Struct Biotechnol J 2016; 4: 211-22.

6. Jiang L, Hong L, Yang W, Zhao Y, Tan A, Li Y. Co-expression network analysis of the IncRNAs and mRNAs associated with cervical cancer progression. Arch Med Sci 2019; 15: 754-64.

7. Gasic V, Stankovic B, Zukic B, et al. Expression pattern of long non-coding RNA growth arrest-specific 5 in the remission induction therapy in childhood acute lymphoblastic leukemia. J Med Biochem 2019; 38: 292-8.

8. Peng Z, Zhang C, Duan C. Functions and mechanisms of long noncoding RNAs in lung cancer. Onco Targets Ther 2016; 9: 4411-24.

9. Xu R, Han Y. Long non-coding RNA FOXF1 adjacent non-coding developmental regulatory RNA inhibits growth and chemotherapy resistance in non-small cell lung cancer. Arch Med Sci 2019; 15: 1539-46.

10. Schmitz SU, Grote P, Herrmann BG. Mechanisms of long noncoding RNA function in development and disease. Cell Mol Life Sci 2016; 73: 2491-509.

11. Fila M, Pawłowska E, Blasiak J. Mitochondria in migraine pathophysiology - does epigenetics play a role? Arch Med Sci 2019; 15: 944-56.

12. Liu X, Wang M, Cui Y. LncRNA TP73-AS1 interacted with miR-141-3p to promote the proliferation of non-small cell lung cancer. Arch Med Sci 2019; 15: 1547-54.

13. Liu H, Zhang Q, Lou Q, et al. Differential analysis of IncRNA, miRNA and mRNA expression profiles and the prognostic value of IncRNA in esophageal cancer. Pathol Oncol Res 2020; 26: 1029-39.

14. Hu X, Bao J, Wang Z, et al. The plasma IncRNA acting as fingerprint in non-small-cell lung cancer. Tumour Biol 2016; 37: 3497-504.

15. Lin Y, Leng $\mathrm{Q}$, Zhan M, Jiang F. A plasma long noncoding RNA signature for early detection of lung cancer. Transl Oncol 2018; 11: 1225-31.

16. Liang W, Lv T, Shi X, et al. Circulating long noncoding RNA GAS5 is a novel biomarker for the diagnosis of nonsmall cell lung cancer. Medicine (Baltimore) 2016; 95: e4608.

17. Tan Q, Zuo J, Qiu S, et al. Identification of circulating long non-coding RNA GAS5 as a potential biomarker for nonsmall cell lung cancer diagnosis, long non-coding RNA, plasma, GAS5, biomarker. Int J Oncol 2017; 50: 1729-38.

18. Williams GT, Mourtada-Maarabouni M, Farzaneh F. A critical role for non-coding RNA GAS5 in growth arrest and rapamycin inhibition in human T-lymphocytes. Biochem Soc Trans 2011; 39: 482-6.

19. Fleming JV, Hay SM, Harries DN, Rees WD. Effects of nutrient deprivation and differentiation on the expression of growth-arrest genes (gas and gadd) in F9 embryonal carcinoma cells. Biochem J 1998; 330: 573-9.

20. Ma C, Shi X, Zhu Q, et al. The growth arrest-specific transcript 5 (GAS5): a pivotal tumor suppressor long noncoding RNA in human cancers. Tumour Biol 2016; 37: $1437-44$.

21. Shi X, Sun M, Liu H, Yao Y, Kong R, Chen F. A critical role for the long non-coding RNA GAS5 in proliferation and apoptosis in non-small-cell lung cancer. Mol Carcinog 2015; 54: E1-12.

22. Wu Y, Lyu H, Liu H, Shi X, Song Y, Liu B. Downregulation of the long noncoding RNA GAS5-AS1 contributes to tumor metastasis in non-small cell lung cancer. Sci Rep 2016; 6: 31093.

23. Dong L, Li G, Li Y, Zhu Z. Upregulation of Long Noncoding RNA GAS5 Inhibits Lung Cancer Cell Proliferation and Metastasis via miR-205/PTEN Axis. Med Sci Monit 2019; 25: 2311-9.

24. Laurent LC, Abdel-Mageed AB, Adelson PD, et al. Meeting report: discussions and preliminary findings on extracellular RNA measurement methods from laboratories in the NIH Extracellular RNA Communication Consortium. J Extracell Vesicles 2015; 4: 26533.

25. Kamel LM, Atef DM, Mackawy AMH, Shalaby SM, Abdelraheim N. Circulating long non-coding RNA GAS5 and SOX2OT as potential biomarkers for diagnosis and prognosis of non-small cell lung cancer. Biotechnol Appl Biochem 2019; 66: 634-42. 